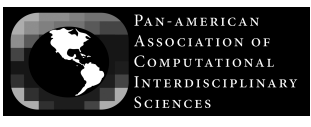

Journal of Computational Interdisciplinary Sciences (2009) 1(2): 141-147

(C) 2009 Pan-American Association of Computational Interdisciplinary Sciences

ISSN 1983-8409

http://epacis.org

\title{
Bio-inspired algorithms applied to microstrip antennas design
}

\author{
Carlos Henrique da Silva Santos ${ }^{1}$, Kleucio Claudio², Marcos Sérgio Gonçalves ${ }^{3}$, \\ Juliano Rodrigues Brianeze ${ }^{4}$ and Hugo Enrique Hernández-Figueroa ${ }^{5}$
}

Manuscript received on November 12, 2008 / accepted on May 4, 2009

\begin{abstract}
This work presents the efficiency and the stochastic nature of three bio-inspired algorithms combining four different probability density functions (PDF) to design Microstrip Antennas (MSAs). The project of MSAs considers single and multi-objective versions. The algorithms examined are Genetic Algorithm (GA), Evolution Strategies (ES) and Artificial Immune Systems (AIS). In these algorithms, bandwidth (BW), standing wave rate (SWR) and radiation efficiency (RE) are parameters to be optimized. The results show interesting computational performance of the algorithms in single and multi-objective applications, combining different PDFs and bio-inspired algorithms. Other interesting result is presented when there is absence of memory resource. These results emphasize the efficiency of the algorithms to optimize MSAs and the possibility to apply in others electromagnetic problems.
\end{abstract}

Keywords: Microstrip Antenna Design, Computational Electromagnetism, Bio-Inspired Algorithms, Multi-objective Optimization.

Correspondence to: Carlos Henrique da Silva Santos

Department of Microwaves and Optics, School of Electrical and Computer Engineering, State University of Campinas (UNICAMP), Brazil, Albert Einstein Avenue, 400.

1E-mail: henrique@dmo.fee.unicamp.br

2E-mail: kleucio@dmo.fee.unicamp.br

3E-mail: brianeze@dmo.fee.unicamp.br

4E-mail: marcoss@dmo.fee.unicamp.br

5E-mail: hugo@dmo.fee.unicamp.br 


\section{INTRODUCTION}

New technologies are emerging increasingly fast [1]. In telecommunications area, this is particularly notable in industrial scale, where there is demand for new and expensive devices.

In order to reduce costs and streamline this process, most of the telecommunication institutions has adopted computational simulators with different optimization tools.

The optimization tools have been very important to provide new devices and solve some complex electromagnetic problems, such as the design of new microstrip antennas for RFID (Radio Frequency Identification) or mobile applications.

This work has two different objectives. At first, to examine three different bio-inspired algorithms applied to optimize the design of microstrip antennas (MSAs). Second, to analyze the stochastic nature of each algorithm in single and multi-objective problems, using four different numerical probability density function (PDF).

The bio-inspired algorithms used are Genetic Algorithm (GA), Evolution Strategies (ES) and Artificial Immune Systems (AIS). These algorithms were chosen because they present different characteristics in the optimization process, specially in the selection criteria of the best candidate solutions.

The Genetic Algorithm and Evolution Strategies are based on Neo-Darwinian Theories. These algorithms are based on recombination, mutation and selection of best individuals. This species evolution is not a pre-defined process and has a stochastic nature.

The main difference between GA and ES is GA describes chromosomes and genes relations while ES algorithms describes the relations between parents and their offspring.

Furthermore, AIS is a simplified computational model of the vertebrate immune system, based on the relations of antigen and antibody to match. The AIS has been recently applied in computational electromagnetism problems [2].

\section{BIO-INSPIRED ALGORITHMS}

This section presents some concepts about Genetic Algorithm (GA), Evolution Strategies (ES) and Artificial Immune System (AIS).

In this work, these three bio-inspired algorithms were developed with $\mathrm{C}_{++}$language and follow the object orientation specifications. This computational resource will be important to reuse components in future works.

\subsection{Genetic Algorithm}

Genetic Algorithm (GA) is the widest bio-inspired algorithm used in Computational Electromagnetism [3]. This algorithm was proposed in 1960's by J. Holland and his collaborators at Michigan University. They would like to analyze the adaptation phenomenon occurred in nature and download this phenomenon for the computers [4].

The GA has a stochastic nature, involving a set of candidate solutions (population) in order to satisfy one or more objective functions (fitness). There are operators to generate population variability and to search best solutions in the computational domain. The main operators are mutation and crossover (recombination). Other important resource is the criterion to select best individuals [5].

This work uses a GA with Gaussian mutation and crossover of single gene. The selection criterion is ranking. Elitism was adopted to guarantee the survival of best individuals.

\subsection{Evolution strategies - $(\mu / \rho, \lambda) E S$ and $(\mu / \rho+\lambda) E S$}

The ES has essential characteristics similar to other evolutionary algorithms, such as Genetic Algorithm and Genetic Programming. However, ES algorithms have different candidate solution representation and selection criteria.

They are represented by $\mu$ parents that are recombined in a group of $\rho$ individuals to generate $\lambda$ offspring.

In each generation the number of individuals in the population varies from $\mu$ to $(\mu+\lambda)$ individuals, and after the selection procedure the population is reduced to $\mu$ individuals again.

The selection is basically defined according to the use of parents and offspring groups. In this context, two of the most recent ES selection are:

- $(\mu / \rho+\lambda) E S$ : This selection criterion considers both groups (parents and offspring) to be selected. The nonselected individuals are removed from the population, storing the best individuals along the generations.

- $(\mu / \rho, \lambda) E S$ : This selection criterion considers only offspring group of each generation to select $\mu$ individuals. For this reason, the amount of individuals in offspring group must be greater or equal than in parent group $(\lambda \geq \mu)$. That absents the use of memory to store best individuals.

Considering these two selection criteria previously presented, with and without memory resources, this work analyzes the importance of them in MSAs optimizations and how their convergences efficient are. 


\subsection{Artificial Immune System - opt-AiNet}

The Artificial Immune System (AIS) can be defined as an adaptive system inspired by theoretical and practice vertebrate immune systems, applied to solve complex computational problems [6].

This technique encapsulates a comprehensive representation of the relationship between antigens and antibodies. But only recently AIS has been explored to solve computational electromagnetic problems [2].

In this work, a version of the optimization artificial immune network algorithm (opt-AiNet) applying Clonal Selection proposed by de Castro [7] is used to design MSAs.

This algorithm ignores the differences between B-cells and antibody, representing them in the same structure. In addition, it is inspired in antibody cloning and affinity process, caused by B-cells clonal expansion, that is changed to match with antigens as quickly as they can.

The matching between antigen and antibody (B-cells) contributes to resize the number of antibodies and to improve the convergence [8].

The most important AIS operator to introduce diversity into the population is the mutation. It is applied to generate different clones and to perform local and global search.

The selection occurs during the suppression procedure. It is a local search executed to measure the affinity between individuals. A pseudo-code of opt-AiNet is presented below [9].

\begin{tabular}{|c|c|}
\hline \\
\hline \multicolumn{2}{|c|}{$\begin{array}{l}\text { while (condition satisfied) } \\
\text { begin }\end{array}$} \\
\hline clone(); & // clone antibody \\
\hline mutation(); & // random changes \\
\hline affinity(); & // antibody affinity \\
\hline $\begin{array}{l}\text { suppression(); } \\
\text { end }\end{array}$ & // select best antibodies \\
\hline
\end{tabular}

This work applies an opt-AiNET with Gaussian mutation and the suppression is based on a golden relation [9].

\section{MICROSTRIP ANTENNAS}

MSAs consist of a metallic thin patch placed a small fraction of the wavelength above the ground plane. The broadside radiation pattern is obtained with an appropriated excitation from below the patch [10].

For a rectangular MSA, the length $L$ of the patch varies usually between $\lambda_{0} / 3$ and $\lambda_{0} / 2$ (where $\lambda_{0}$ is the wavelength in free space).

The metallic patch and the ground plane are separated by a dielectric layer, the substrate. There are many kinds of substrates to be used in MSAs, with different values for dielectric constant. The patch may have several forms, the most common ones being the rectangular and the circular, for their easy analysis and manufacture and their attractive radiation features.

There are many possible configurations to feed a MSA. The four more popular are by microstrip line, by coaxial probe, by aperture coupling, and by proximity coupling [10].

This work considers only MSAs with coaxial probe feeding. In this case, the internal conductor is connected to the patch, while the external is connected to the ground plane. With this kind of feeding, the desired input impedance may be obtained by appropriately placing the internal conductor.

The optimized parameters from a rectangular MSA, except dielectric constant of substrate, are shown in Figure 1, where $x_{i n}$ is the distance from the feed point to the nearest resonant side, $L$ is the non-resonant side length, $W$ is the resonant side length, and $h$ is the substrate height.

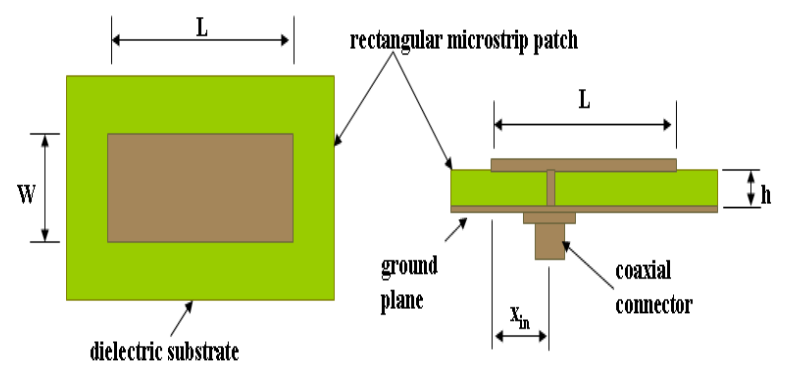

Figure 1 - The MSAs Parameters to be optimized.

In the simulations it was imposed that:

1. The value of $h$ is limited by the interval of $0.1 \mathrm{~mm}$ to $10 \mathrm{~mm}$;

2. The relative dielectric constant $\left(\varepsilon_{r}\right)$ of substrate is limited to the range 1 to 10 ;

3. To obtain an MSA for $2.4 \mathrm{GHz}, W$ and $L$ are limited between 7 and $4 \mathrm{~cm}$;

4. As input impedance is symmetrical around the centre of the patch, $x_{i n}$ cannot be greater than $L / 2$; it should therefore be smaller than $3.5 \mathrm{~cm}$, and any solution with $x_{i n}>L$ must be eliminated.

A CAD procedure, based on equations derived from the Cavity Method, is used to calculate the antenna's several parameters [11].

The validity of the expressions to the parameters was previously guaranteed to all the situations considered. One of the MSA configurations obtained was also simulated in commercial electromagnetic simulation software [12] for verification. The results for some antenna parameters are compared in Table 1. 
Table 1 - Comparison between some MSA parameters calculated by the CAD Model based on the Cavity Method, and obtained by simulation in commercial EM software. $f_{0}$ is resonant frequency, $D$ is directivity.

\begin{tabular}{|c|c|c|}
\hline MSA parameters & CAD model & Commercial EM software \\
\hline SWR (minimum) & 1.72268 & 1.544 \\
\hline $\mathrm{f}_{0}(\mathrm{GHz})$ & 2.40563 & 2.326 \\
\hline $\mathrm{BW}(\%)(\mathrm{S} 11 \leq-6 \mathrm{~dB})$ & 6.30184 & 2.49 \\
\hline $\mathrm{RE}(\%)$ & 96.6614 & 100 \\
\hline $\mathrm{D}(\mathrm{dB})$ & 6.07199 & 8.059 \\
\hline
\end{tabular}

One can notice in Table 1 that some parameters received different values. The greater differences occur for BW and D. This was not expected and diminishes the credibility of the modelling based on the Cavity Method. It however does not devaluate the evolutionary optimization and the comparison among the methods.

\section{MICROSTRIP ANTENNAS OPTIMIZATION: PROCEDURES AND RESULTS}

All bio-inspired algorithms use random numbers in their working mechanism, mainly at the operators. The random numbers can be generated with different probability density functions (PDFs), resulting in different effects in the algorithms, making them more appropriate to solve different problems.

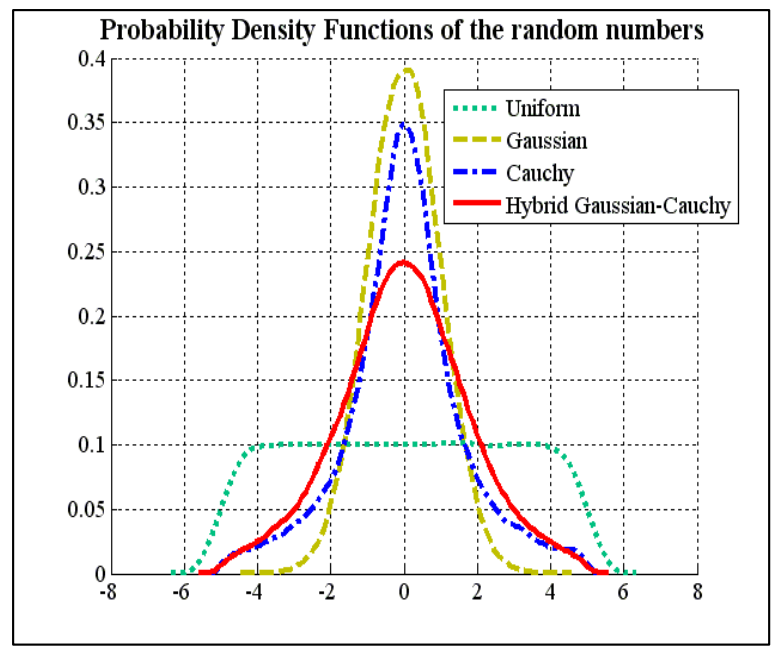

Figure 2 - Probability density functions of the random numbers used in the algorithms, with $\mu=0, \sigma^{2}=1$ and limited between -6 and 6 .

In bio-inspired algorithms implemented in this work, the effect of random numbers with four different PDFs is assessed. Their PDFs are approximately Uniform, Gaussian, Cauchy or a Hybrid Gaussian-Cauchy, as shown in Figure 2.
In the hybrid case, the random number is a linear combination of a Gaussian and a Cauchy random number, given by

$$
H\left(\mu, \sigma^{2}\right)=N\left(\mu, \sigma^{2}\right)+\beta \cdot C\left(\mu, \sigma^{2}\right)
$$

where $N\left(\mu, \sigma^{2}\right)$ is a Gaussian random number with mean $\mu$ and variance $\sigma^{2}, C\left(\mu, \sigma^{2}\right)$ is a Cauchy random number with median $\mu$ and scale parameter $\sigma^{2}$, and $\beta$ is the ratio between both random numbers in the combination.

Each bio-inspired algorithm implements individuals represented by a vector with the value of the variables to be optimized: $I=\left[x_{i n}, L, W, h, \varepsilon_{r}\right]$. In all performed tests, the total number of generations is 1000 and the frequency desired is $2.4 \mathrm{GHz}$, with acceptable limit error of $5 \%$.

The chromosomes in the population are always initialized with uniform random values, between the limits -5 and 5 . During the following generations these values are altered by the genetic operators. Before each fitness evaluation, these chromosome values are converted to a value between the limits of each variable under optimization in the MSA and proportional to its previous position in the range $[-6,6]$.

The following optimization targets are adopted: to minimize the standing wave ratio (SWR), and to maximize the radiation efficiency (RE) and bandwidth (BW), besides to tune the antenna to $50 \Omega$ input impedance and to the desired operational frequency. This is, therefore, a multi-objective optimization.

Whenever a variable $X$ reaches a value beyond its maximum or minimum allowed limits, through the genetic operators, it is adopted a repair procedure. The variables then receive a value rep $_{1}=X_{\max }-N$ or rep ${ }_{2}=X_{\min }+N$, respectively, if the maximum or minimum limit is exceeded, where $N$ is a uniform random number between the variable limits.

Each EA has other specific features as well, which will be detailed next.

\subsection{Single objective approach}

To turn the MSA optimization problem into a single objective problem (the simplest approach to multiobjective problems), the fitness function $F$ was empirically adopted:

$$
F=\frac{e_{r}+(1 / S W R)+20 \times B W+F_{1}}{4 \cdot F_{2}}
$$

where

$$
F_{1}=1-\frac{\left|50-R_{i n}\right|}{50}
$$

if $\left|50-R_{i n}\right| \leq 50$ or $F_{1}=0$, and $F_{2}=0$ if $f_{0}<$ $f-(f \cdot d f) / 2$ or $f_{0}>f+(f \cdot d f) / 2$, or $F_{2}=1$ otherwise. 
Each of the elements added in Equation (1) receives an approximate equal weight. The use of $F_{2}$ imposes a penalty to the individual whose $f_{0}$ is out of the supplied acceptable percent error, and $F_{1}$ reinforces the objective of minimizing SWR.

Each normalized convergence analysis of the bio-inspired algorithms was executed 10 times and the results presented below are the average of these executions. The values above 0.9 in average are considered successful results.

In Figure 3 it is shown the convergence efficiency of each bio-inspired algorithm to design MSAs using a Uniform Probability Density Function. In this case, GA presents the best convergence solution in average.

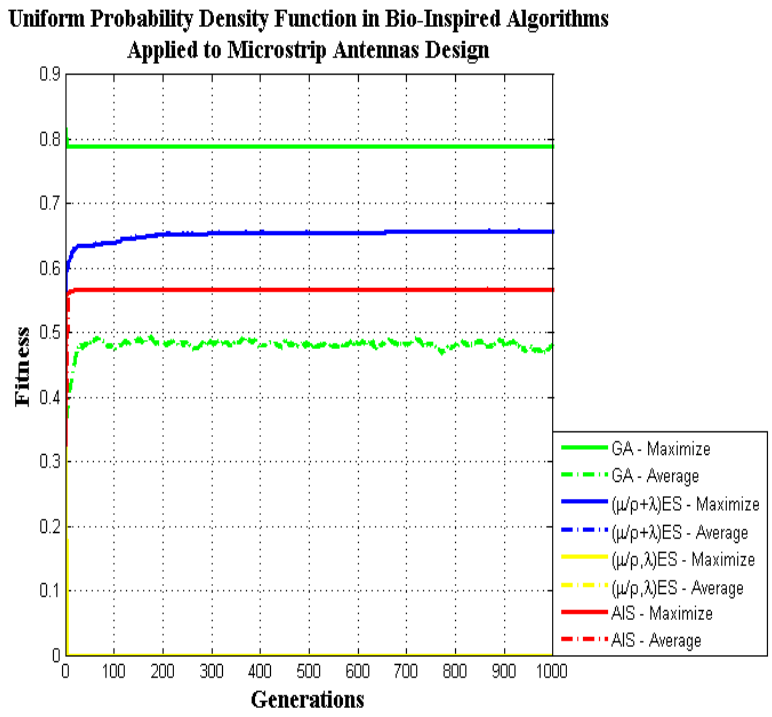

Figure 3 - MSA design applying Uniform PDF.

In Figure 3 one can also notice the non convergence of the $(\mu / \rho, \lambda) E S$. It is motivated by the absence of memory resource.

The second benchmark test applies a Gaussian Probability Function to design MSAs. In the average, $(\mu / \rho+\lambda) E S$ is the best solution and it achieves better results than GA in the first test. In this second PDF analysis, $(\mu / \rho, \lambda) E S$ suffered major variations over the generations, but it does not converge again.

The next MSA design with Cauchy PDF (Fig. 5) presents better results than the first and second PDF tests. The $(\mu / \rho+\lambda) E S$ algorithm achieves the maximum value expected near 800 generations and GA also converges to successful results.

In the last test using a Hybrid Gaussian-Cauchy PDF (Fig. 6) the efficiency convergence was not good. All algorithms did not converge for successful results in 1000 generations, as expected.

The AIS presents stagnation in all tests. It should be better analyzed using other operators to reach efficient convergence.

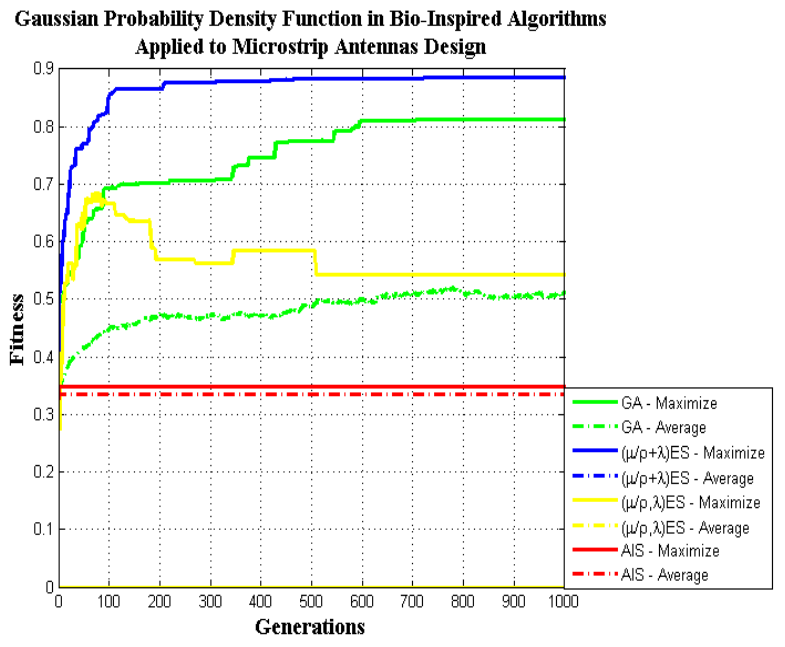

Figure 4 - MSAs design applying a Gaussian PDF.

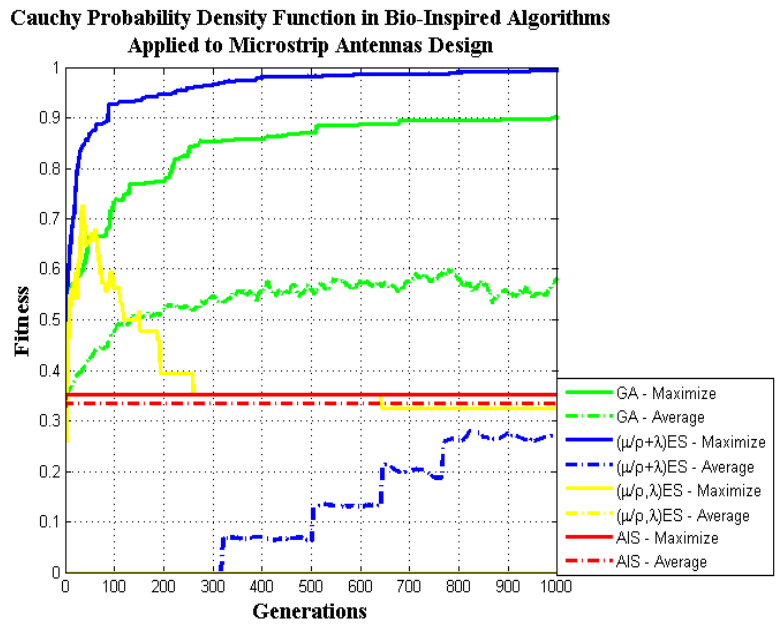

Figure 5 - MSAs design applying a Cauchy PDF.

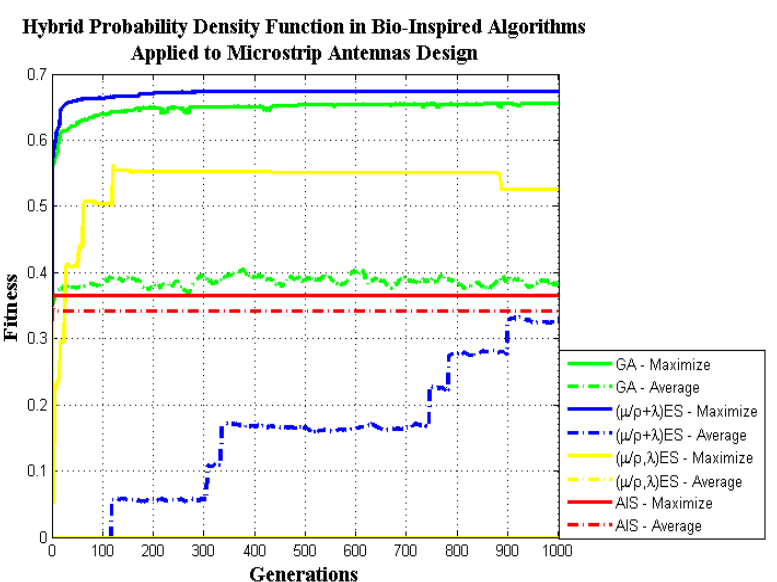

Figure 6 - MSAs design applying a Hybrid Gaussian-Cauchy PDF. 


\subsection{Multi-objective approach}

The project of new devices in telecommunications generally requires that several parameters are known. It is an example of multi-objective problems and it also happens with most projects of antennas.

For this reason, this work presents the convergence efficiency of the bio-inspired algorithm with four different Probability Density Functions. The Pareto Frontier is obtained by the average of 10 trials.

All bio-inspired algorithms in multi-objective version apply Pareto Frontier technique. In this case, the individuals that achieve best results, satisfy all conditions and in which there are not best individuals with similar characteristics are called dominate and assumed to be in Pareto Frontier.

When an individual do not satisfy all fitness conditions or there is at least one master individual, it is called dominate and out of Pareto Frontier.

In Figure 7 it can be verified the Pareto Frontier obtained from the MSA design, applying Uniform Probability Density Function.

Uniform Probability Density Function for Multiobjective Pareto Frontier Applied to Microstrip Antenna Design

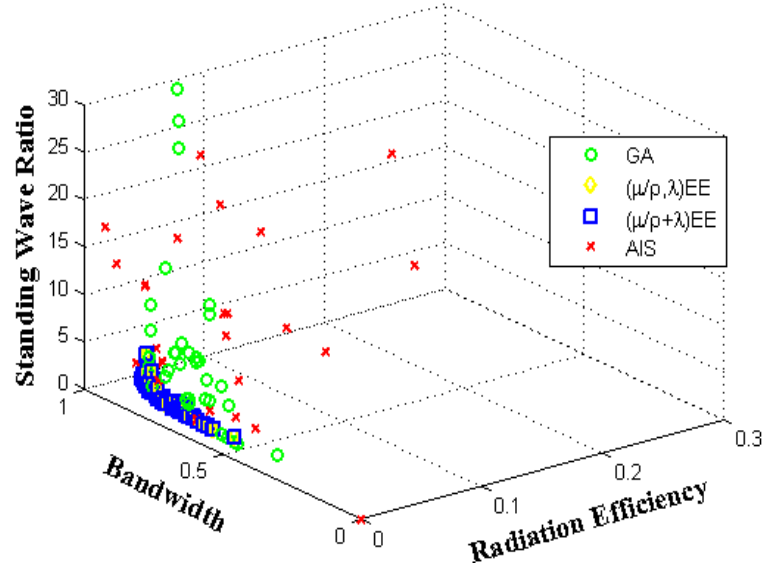

Figure 7 - Pareto Frontier of the MSA design with Uniform PDF

In Figure 7 it is possible to note the grouping of $(\mu / \rho+$ $\lambda$ ) $E S$ candidate solutions. GA and AIS present more sparse individuals, meaning that the search area was further explored. In addition, GA presents some similarities with $(\mu / \rho+\lambda) E S$ in the main region. In this case, GA with Uniform PDF presents the best solution.

In Figure 8 it is presented the multi-objective MSA design applying Gaussian PDF.

The main difference between the algorithms occurs in GA, which presents more sparse individuals again and a lot of individuals in the main region. This Gaussian PDF improves the similarities in the algorithms and the convergence efficiency of each one.

\section{Gaussian Probability Density Function for Multiobjective Pareto Frontier Applied to Microstrip Antenna Design}

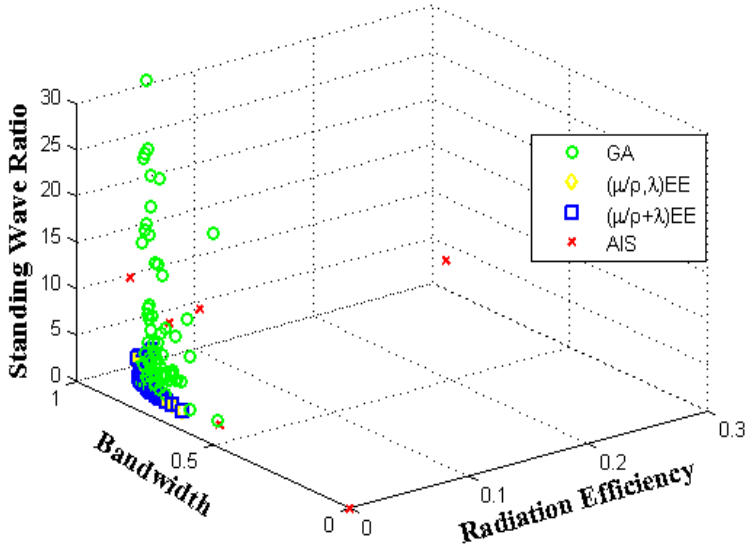

Figure 8 - Pareto Frontier of the MSA design with Gaussian PDF.

Cauchy Probability Density Function for Multiobjective Pareto Frontier Applied to Microstrip Antenna Design

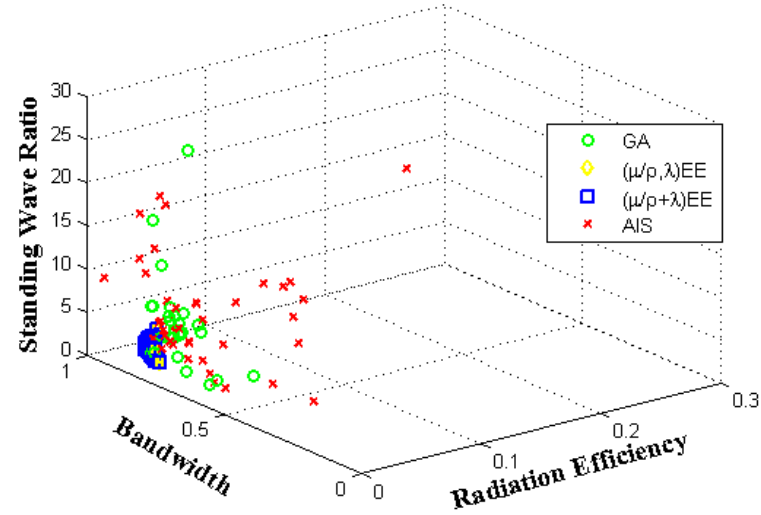

Figure 9 - Pareto Frontier of MSA design applying Cauchy Probability Density Function.

Hybrid Probability Density Function for Multiobjective Pareto Frontier Applied to Microstrip Antenna Design

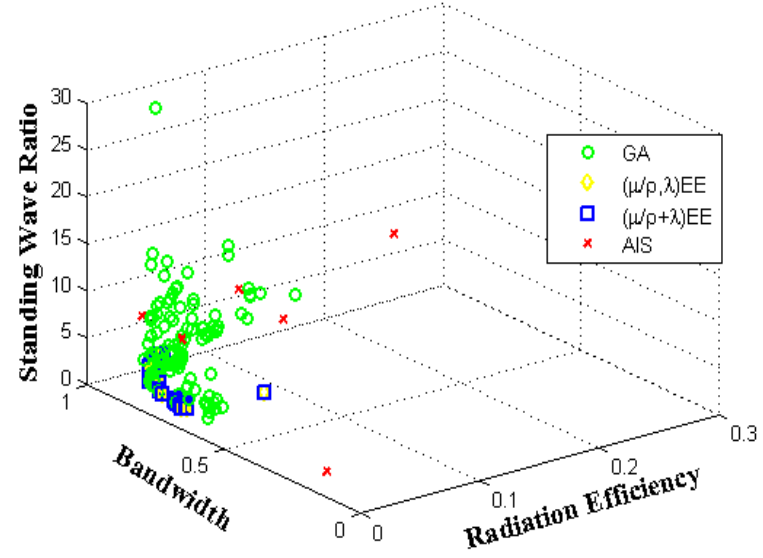

Figure 10 - Pareto Frontier of MSA design applying Hybrid PDF. 
The importance of analyzing different PDF in bio-inspired algorithms is perceived in single and multi-objective problems, because the convergence efficiency of the bio-inspired algorithms is different according to the PDF adopted. Figures 9 and 10 show this behavior in MSA multi-objective design applying Cauchy and Hybrid (Cauchy-Gaussian) PDF.

The ES algorithms present similar results in multi-objective tests. The AIS presents some individuals out of Pareto Frontier, and should be reviewed for multi-objective applications.

\section{CONCLUSIONS}

The results obtained in single and multi-objective MSA design evidence the impact of probability density function in bio-inspired algorithms.

In single objective, the best solution to design MSAs is the combination of Cauchy PDF and $(\mu / \rho+\lambda) E S$ algorithm, which achieves the best efficiency convergence and maximum normalized values.

In multi-objective optimization the best candidate solutions in Pareto Frontier are presented by GA with Gaussian PDF. This work shows different combinations of bio-algorithms and PDF to solve single and multi-objective problems. It is important to emphasize the stochastic nature of the algorithms.

Another important result to highlight is the unstable behaviour of the $(\mu / \rho, \lambda) E S$ in single objective problems. It occurs due to the absence of memory resource.

The AIS should be reviewed in single and multi-objective versions. In addition, new operators would be applied in these bioinspired algorithms to examine the convergence efficiency.

Future works are considering the integration of numerical methods, such Finite Element Method (FEM) and Finite Difference Time Domain (FDTD) method, to project more complex devices. In this case, a parallel version of these bio-inspired algorithms will be applied.

\section{ACKNOWLEDGMENTS}

The authors wish to acknowledge the assistance and support of FAPESP (Process 06/57074-2), CAPES and Laboratory of Applied and Computational Electromagnetism (LEMAC), from State University of Campinas (UNICAMP).

\section{REFERENCES}

[1] ABRANTES AA \& MARTINS LM. 2007. A produção do conhecimento científico: relação sujeito-objeto e desenvolvimento do pensamento. Interface Comunicações, 11: 313-325.

[2] CAMPELO F, GUIMARÃES FG, IGARASHI H \& RAMÍREZ JA. 2005. Clonal Selection Algorithm for Optimization in Electromagnetics. IEEE Transactions on Magnetics, 41(5): 1736-1739.

[3] HAUPT RL \& WERNER DH. 2007. Genetic Algorithms In Electromagnetics. John Wiley \& Sons, New Jersey.

[4] MITCHELL M. 1998. An Introduction to Genetic Algorithms. The MIT Press, Massachusetts.

[5] BRECKLING J (Ed.). 1989. The Analysis of Directional Time Series: Applications to Wind Speed and Direction, ser. Lecture Notes in Statistics. Berlin, Germany: Springer, vol. 61.

[6] DE CASTRO LN. 2006. Fundamentals of Natural Computing Basic Concepts, Algorithms, and Applications. Chapman \& Hall/CRC, Florida.

[7] DE CASTRO LN \& TIMMIS J. 2002. Artificial Immune Systems: A New Computational Intelligence Approach, Springer-Verlag, Great Britain.

[8] LIU F, WANG Q \& GAO X. 2004. Survey of Artificial Immune Systems. IEEE International Conference on System, Man and Cybernetics, 4: $3415-3420$.

[9] FRANÇA F0. 2005. Algoritmos Bio-Inspirados Aplicados a Otimização Dinâmica. M.Sc. Thesis, School of Electrical and Computer Engineering, Unicamp, Campinas,

[10] BALANIS CA, 2005. "Antenna Theory: Analysis and Design", John Wiley \& Sons, Inc.

[11] LEE KF, CHEN W. 1997. Advances in Microstrip and Printed Antennas. J. Wiley \& Sons, New York.

[12] CST Microwave Studio 2006B, www.cst.com (accessed in March, 2008). 